\title{
EGRET Gamma-Ray Observations of the Crab P2/P1 Ratio
}

\author{
W. F. Tompkins ${ }^{1}$, B.B. Jones, P.L. Nolan \\ W. W. Hansen Experimental Physics Laboratory, Stanford University, Stanford CA 94305 \\ G. Kanbach \\ Max Planck Institut für Extraterrestische Physik, D85748 Garching, Germany \\ and \\ P. V. Ramanamurthy, D. J. Thompson \\ NASA/Goddard Space Flight Center, Code 661, Greenbelt MD 20771
}

\begin{abstract}
Recent observations of the Crab pulsar by the Energetic Gamma-Ray Experiment Telescope (EGRET) on the Compton Gamma Ray Observatory show that the highenergy gamma-ray light curve has changed little over the lifetime of the instrument. Previous data collected by SAS-2 and COS-B in the years 1972-82, along with earlier EGRET data, suggested a 14 year sinusoidal variation in the flux ratio between the first and second peaks. The new data from EGRET indicate that the flux ratio is constant.
\end{abstract}

Subject headings: gamma-rays: observations - pulsars : individual (Crab)

Accepted Astrophysical Journal. Scheduled 20 Sep 1997.

${ }^{1}$ billt@egret0.stanford.edu 


\section{Introduction}

High energy gamma ray emission from the Crab pulsar was observed by satellite-borne telescopes for 15 years: in 1972-73 by SAS-2 (Kniffen et al. 1974), from 1975-82 by COS-B (Clear et al. 1987), and since 1991 by EGRET (Nolan et al. 1993; Ramanamurthy et al. 1995). Early observations showed possible sinusoidal variation in the relative intensities of the two peaks (Wills et al. 1982) with a time scale of $\sim 14$ years, and it was suggested that this variation might be due to the precession or free nutation of the neutron star (Kanbach 1990, Özel 1991). An apparent confirmation of the sinusoidal signal was seen in the low energy gamma ray emission (Ulmer et al. 1994), matched in phase and period with the high energy results, but with a smaller amplitude. EGRET data from 1991 through early 1994 were consistent with the expected variation (Nolan et al. 1993, Ramanamurthy et al. 1995), although these observations spanned a time when the ratio of the peaks was predicted to be fairly constant, near the minimum of the sinusoid.

EGRET observations have now extended the available data by over two years. The most recent data were expected to be $4-6 \sigma$ from the average of the previous values if the sinusoidal model is correct.

\section{Observations and Analysis}

The EGRET instrument is a spark chamber gamma-ray telescope with an energy range of $30 \mathrm{MeV}-30 \mathrm{GeV}$. Details of the instrument design, calibration, and standard analysis software are given in Thompson et al. (1993).

All viewing periods where EGRET was pointed within $20^{\circ}$ of the Crab were analyzed, with the exception of viewing period 0021 (1991 Jul 815), in which there was a large solar flare. The Compton Observatory viewing period numbers and dates for these observations are shown in Table 1 . The eight viewings numbered 4120 through 5280 (1995 Feb. through 1996 Aug.) have been completed since the time of the previous work of Ramanamurthy et al. (1995).

All photons with measured energy above 50 $\mathrm{MeV}$, which were within an energy-dependent cone of half-angle $\theta_{\max }$ were used in this analysis. The angle $\theta_{\max }$, chosen such that $68 \%$ of the photons originating from the pulsar are within the acceptance cone, is given by (Thompson et al. 1993)

$$
\theta_{\max }=5^{\circ} .85 \times(E / 100 \mathrm{MeV})^{-0.534} .
$$

The arrival time of each detected photon was transformed to Solar System Barycentric Time using the DE200 ephemeris, then binned according to the pulsar phase at that time, determined from the Princeton Pulsar Timing Database (Arzoumanian et al. 1992). This analysis was performed using the PULSAR program (Fierro 1995).

As seen in Figure 1, the light curve was divided into several sections, including Peak 1 (phase .94 - .04), Peak 2 (phase .32-.46), and the off-pulse Background (phase .46-.94). These definitions follow those used in the COS-B analysis (Wills et al. 1982) and are similar to those used by Nolan et al. (1993) and Ramanamurthy et al. (1995). The background (from the Crab nebula, nearby sources, and the diffuse Galactic radiation) was assumed constant as a function of pulsar phase. The off-pulse count rate was then used to find the background-subtracted counts estimates of the two peaks ( $P 1$ and $P 2)$. In order to avoid any effects of changes in instrument performance, the evolution of the ratio $P 2 / P 1$ was examined (as in previous analyses).

The two peaks have slightly different energy spectra (Nolan et al. 1993), and the EGRET response at different energies has changed at different rates (Esposito et al. 1997). Thus the ratio $P 2 / P 1$ is affected by the changes in instrument performance over time. Calculations of this effect, however, indicate that it is an order of magnitude smaller than the errors in $P 2 / P 1$ due to Poisson fluctuations. 

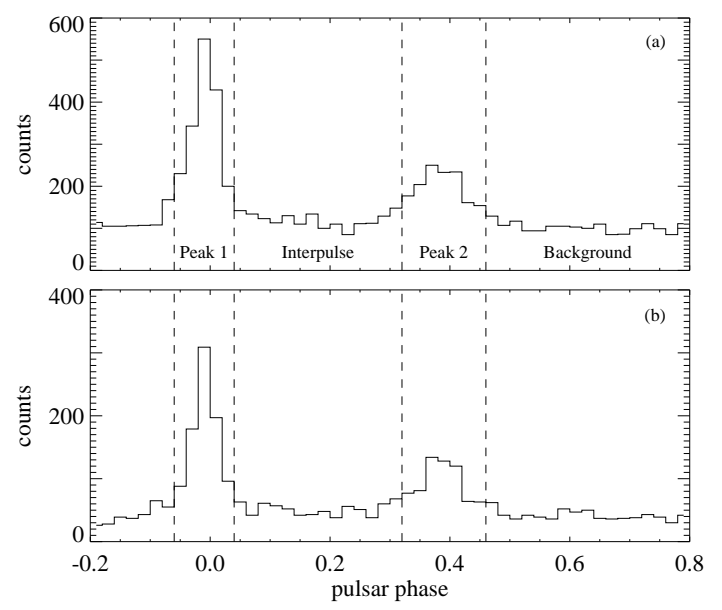

Fig. 1.- Gamma-ray phase histograms of photons with $E>50 \mathrm{MeV}$ for EGRET observations of the Crab in 1991$2(a)$ and 1995-6 (b). The vertical dashed lines indicate phase boundaries used in the peak height analysis. The horizontal dashed lines indicate the background level as determined from the data.

The differences in the energy responses of the SAS-2, COS-B, and EGRET instruments are larger than the variation in the EGRET response. However, statistical errors in the previous instruments' data are larger as well. Thus the value of $P 2 / P 1$ obtained from EGRET data should be comparable to that obtained with SAS2 and COS-B.

\section{Results}

The values of $P 2 / P 1$ obtained, together with the $1 \sigma$ errors, are shown in Table 1 . A reduced data set, where nearby points are joined for clarity, is shown in Figure 2. The data were fit with a constant, yielding $P 2 / P 1=.54 \pm .03$, with $\chi^{2}=6.01$ with 20 degrees of freedom (DOF). Such a low value of $\chi^{2}$ might imply that the errors in the data were over-estimated. In this case, however, the errors arise purely from statistical Poisson fluctuations, and the low value must occur purely by chance. The data are very consistent with a constant value of $P 2 / P 1$. The data were also fit with a straight line $\left(\chi^{2}=5.96\right.$ with

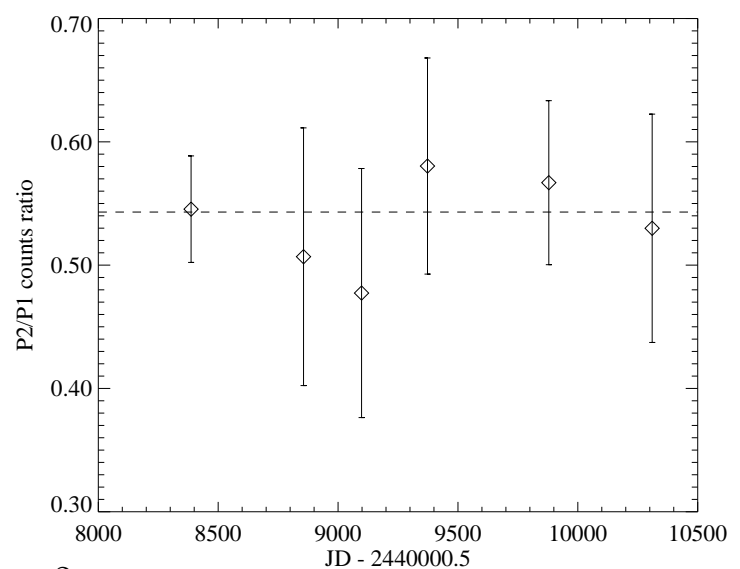

Fig. 2.- Variation in the ratio of the two peaks in the Crab light curve for $E>50 \mathrm{MeV}$ (from Table 1). For clarity, the 21 observations are grouped into 6 data points, where each point represents the average of several nearby observations. Error bars are $1 \sigma$. The dashed line is the average of all EGRET observations.

19 DOF $)$, and a quadratic $\left(\chi^{2}=5.92\right.$ with 18 DOF). Neither result gives a significantly better fit: the EGRET data are most consistent with no variation.

The EGRET data were also analyzed in conjunction with the SAS-2 (Kanbach 1990) and COS-B (Clear et al. 1987) data. The best fit sinusoid to the previous instruments' data,

$P 2 / P 1=0.85-0.56 \sin (2 \pi(T-1975.67) / 13.3)$,

where $\mathrm{T}$ is the year of the observation, and to the combined data set,

$P 2 / P 1=0.544-0.060 \sin (2 \pi(T-1976.48) / 11.55)$

are shown together with the data and the average value of $\mathrm{P} 2 / \mathrm{P} 1$ in Figure 3.

As can be seen, the most recent EGRET observations (the last data point) are much less consistent with the large amplitude sinusoid. The constant value of $0.528 \pm 0.027$ gives a $\chi^{2}=24.0$ (with $27 \mathrm{DOF}$ ), indicating a good fit. The sinusoid fit to the combined data gives a period of 11.6 years with $\chi^{2}=21.2$ (with 24 DOF), which does not represent a significant improvement. Thus, using the combined data sets, the 


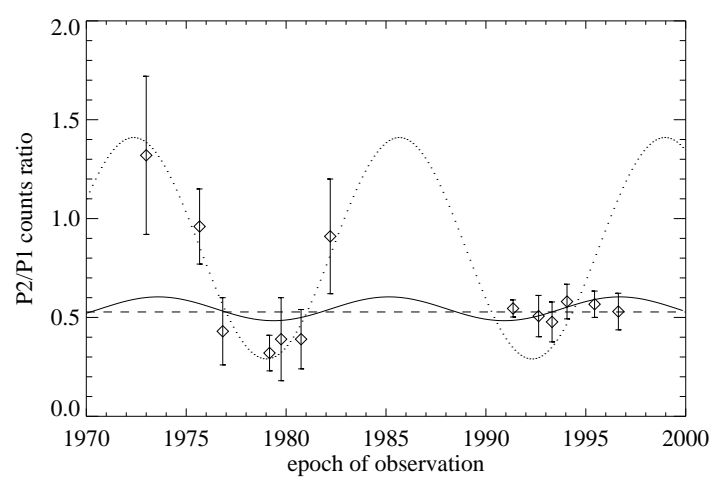

Fig. 3.- Variation in the ratio of the two peaks in the Crab light curve from SAS-2 (1973), COS-B (1975-1983), and EGRET (1991-96), where the EGRET data set has been reduced as in Fig 2. The dotted line is the best fit sinusoid to the pre-EGRET data. The solid line is the best fit sinusoid to all the data. The dashed line is the average of all the data.

data are most consistent with no variation in $\mathrm{P} 2 / \mathrm{P} 1$.

The light curves obtained from Phase 1 data (Apr 91-Sept 92) and from Phases 4 and 5 (Feb 95-Aug 96) are shown in Figure 1. The overall shape seems to have changed little, in contrast with the expected change if the 14 year cycle were correct. A $\chi^{2}$ test was performed to compare the two light curves in a quantitative way. In order to take out possible systematic effects, both an additive offset and a multiplicative factor for the second light curve were fit to the data. The resulting $\chi^{2}=58.5$ (with $48 \mathrm{DOF}$ ) is consistent with no change in the light curve. Without the offset, $\chi^{2}=87.9$ (with 49 DOF) was obtained, indicating an inconsistency at the $99.95 \%$ confidence level. The offset required indicates a lower background level in the later observations. This could be due to a change in the nebular emission (de Jager et al. 1996), or could be a result of changes in the performance of EGRET. As the gas in the spark chamber ages, the sensitive area at low energies decreases, which decreases the width of the average point spread function. This effect might lower the background in the later observations.

\section{Summary and Conclusion}

Recent observations with EGRET have provided data relevant to the reported variation in the $\mathrm{P} 2 / \mathrm{P} 1$ ratio of the Crab pulsar. Data from SAS-2 and COS-B suggested a sinusoidal variation in this ratio. The EGRET data, both taken alone and in conjunction with the data from previous instruments, are most consistent with a constant value of $\mathrm{P} 2 / \mathrm{P} 1$. Examination of the light curves from early and later observations shows no distinct changes in the pulsar's light curve. The EGRET data cannot, of course, rule out past variability in the $\mathrm{P} 2 / \mathrm{P} 1$ ratio. Future observations by EGRET and its successors may allow a more precise characterization of the long term behavior of the Crab's light curve.

The EGRET team gratefully acknowledges support from the following: The ARCS Foundation (WFT), Bundesministerium für Bildung, Wissenschaft, Forschung und Technologie grant 50 QV 9095 (MPE), NASA Cooperative Agreement NCC 5-95 (HSC), NASA Grant NAG51605(SU), and NASA Contract NAS5-96051 (NGC). 


\section{REFERENCES}

Arzoumanian. Z., Nice, D., \& Taylor, J.H. 1992, GRO/radio timing data base, Princeton University

Clear, J. et al. 1987, A\&A, 174, 85

Esposito, J.A. et al. 1997, in preparation.

de Jager, O.C. et al. 1996, ApJ, 457, 253

Fierro, J. M. 1995, Ph.D. Thesis, Stanford University

Kanbach, G. 1990, in The EGRET Scinece Symposium: NASA Conference Publication No. 3071 ed. Fichtel, C.E. et al. 101

Kniffen, D.A. et al. 1974 Nature, 251397

Nolan, P.L. et al. 1993, ApJ, 409, 697

Özel, M.E. 1991, Europhysics Letters, 14, 3

Ramanamurthy, P.V. et al, 1995, ApJ, 450, 791

Thompson, D.J. et al. 1993, ApJS, 86, 629

Ulmer, M.P. et al. 1994, ApJ, 432, 228

Wills, R.D. et al. 1982, Nature, 296, 723

This 2-column preprint was prepared with the AAS LATEX macros v4.0. 
TABLE 1

Crab P2/P1 for Each EGRET Observation

\begin{tabular}{lrc}
\hline \hline Viewing Period & Dates & P2/P1 \\
\hline 0002 & Apr 22-28 1991 & $0.57 \pm 0.11$ \\
0003 & Apr 28-May 1 1991 & $0.54 \pm 0.15$ \\
0004 & May 1-4 1991 & $0.65 \pm 0.18$ \\
0005 & May 4-7 1991 & $0.55 \pm 0.14$ \\
0010 & May 16-30 1991 & $0.52 \pm 0.07$ \\
0360 & Aug 11-12 1992 & $0.66 \pm 0.34$ \\
0365 & Aug 12-20 1992 & $0.37 \pm 0.15$ \\
0390 & Sep 1-17 1992 & $0.65 \pm 0.17$ \\
2130 & May 23-29 1993 & $0.45 \pm 0.19$ \\
2210 & May 13-24 1993 & $0.49 \pm 0.12$ \\
3100 & Dec 1-13 1993 & $0.55 \pm 0.16$ \\
3211 & Feb 8-15 1994 & $0.59 \pm 0.11$ \\
3215 & Feb 15-17 1994 & $0.61 \pm 0.31$ \\
4120 & Feb-Mar 7 1995 2819.15 \\
4130 & Mar 7-21 1995 & $0.52 \pm 0.15$ \\
4200 & May 23-Jun 6 1995 & $0.58 \pm 0.12$ \\
4260 & Aug 8-22 1995 & $0.45 \pm 0.15$ \\
5020 & Oct 17-31 1995 & $0.50 \pm 0.20$ \\
5260 & Jul 30-Aug 13 1996 & $0.73 \pm 0.14$ \\
5270 & Aug 13-20 1996 & $0.50 \pm 0.12$ \\
5280 & Aug 20-27 1996 & $0.69 \pm 0.21$ \\
& & $0.48 \pm 0.22$ \\
\hline
\end{tabular}

